\title{
De la voix autoritaire à la voix autorisée
}

Les tensions de la narration dans les Manuscrits de Pauline

Archange

From the Authoritarian Voice to the Authorized Voice. Tensions of Narration in Manuscrits de Pauline Archange De la voz autoritaria a la voz autorizada. Llas tensiones de la narración en Manuscrits de Pauline Archange [manuscritos de Pauline Archange]

\section{Stéphane Larrivée et Andrée Mercier}

Volume 37, numéro 1 (109), automne 2011

Marie-Claire Blais

URI : https://id.erudit.org/iderudit/1006463ar

DOI : https://doi.org/10.7202/1006463ar

\section{Aller au sommaire du numéro}

\section{Éditeur(s)}

Université du Québec à Montréal

\section{ISSN}

0318-9201 (imprimé)

1705-933X (numérique)

Découvrir la revue

Citer cet article

Larrivée, S. \& Mercier, A. (2011). De la voix autoritaire à la voix autorisée : les tensions de la narration dans les Manuscrits de Pauline Archange. Voix et Images, 37(1), 73-86. https://doi.org/10.7202/1006463ar
Résumé de l'article

Cet article aborde la trilogie de Marie-Claire Blais, Manuscrits de Pauline Archange, sous l'angle de l'autorité narrative, c'est-à-dire des modalités de représentation et de fonctionnement de la voix narrative ainsi que des enjeux de légitimation qu'elle engage. La notion est d'autant plus intéressante que les Manuscrits de Pauline Archange déploient un vaste réseau thématique autour de l'autorité. Une telle perspective conduit à dégager les contradictions d'un récit porté par une voix autoritaire, fragilisée toutefois par le discours et le jugement de l'autre, et à montrer, dans un deuxième temps, que les tensions qui marquent la narration trouvent leur résolution dans les romans ultérieurs, tels Le sourd dans la ville, Visions d'Anna et, plus tard encore, le cycle inauguré par Soifs. Il ressort que, de la voix autoritaire à la voix autorisée, les Manuscrits de Pauline Archange mettent en scène la difficile conquête de légitimité de l'écriture, à comprendre comme l'atteinte d'une autorité positive de la voix narrative. 


\title{
DE LA VOIX AUTORITAIRE À LA VOIX AUtorisée
}

\author{
Les tensions de la narration
}

dans les Manuscrits de Pauline Archange* 1

$$
+++
$$

\section{STÉPHANE LARRIVÉE}

Centre de recherche interuniversitaire sur la littérature et la culture québécoises (CRILCQ) Université Laval/Université Rennes 2

\section{ANDRÉE MERCIER}

Centre de recherche interuniversitaire sur la littérature et la culture québécoises (CRILCQ) Université Laval

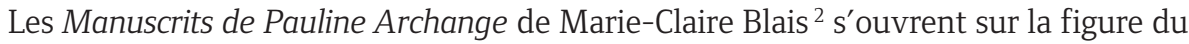
chœur, «le chœur de mes lointaines misères» (MPA, 9) comme se le rappelle la narratrice, celui des religieuses dont les voix s'unissent pour mépriser les mauvais élèves: «Pauline Archange a désobéi, nous la priverons des restes de notre pain d'hostie...» (MPA, 10) Parcours d'apprentissage, les Manuscrits de Pauline Archange le sont à plus d'un titre, car s'ils racontent la difficile traversée de l'enfance d'une petite fille et sa venue graduelle à l'écriture, ils se présentent aussi comme un récit qui cherche à poser sa voix. Dans un monde où le jugement d'autrui se montre omnipotent et son pouvoir le plus souvent violent, Pauline affirme aller son chemin, "défiant une autorité qu'[elle] juge monstrueuse» (MPA, 18). Ce désir d'émancipation se heurte toutefois à de multiples obstacles, dont ce «chœur de fauves amers» (MPA, 41) - religieuses, parents, adultes - qui ne cesse de se faire entendre et de la dominer : «la Pauline, eh ben, elle n'a pas de cœur» (MPA, 18). En tant que narratrice, Pauline souffre de cette «souveraineté brutale» puisque son propre discours se voit pénétré par la parole vindicative de l'autre; or sa voix se montre elle-même autoritaire, alors qu'elle multiplie les sentences sévères, qu'elle fouille les consciences ou ironise aux dépens de certains personnages.

Dans le cadre de cet article, il s'agira donc, dans un premier temps, d'observer la trilogie sous l'angle de l'autorité narrative, notion qui désigne aussi bien les modalités de représentation et de fonctionnement de la voix narrative que les enjeux de

$$
+++
$$

* Pour obtenir la liste des sigles utilisés dans cet article et les références complètes aux œuvres de Marie-Claire Blais, voir p. 7. 1 Cet article s'inscrit dans les suites de deux projets de recherche subventionnés par le Conseil de recherches en sciences humaines du Canada, codirigés par Frances Fortier et Andrée Mercier et ayant pour titre "Autorité narrative et vraisemblance dans le roman contemporain », de même que «Narration impossible, indécidable et ambiguë. Enjeux esthétiques et théoriques de la transmission narrative dans le roman contemporain ». 2 Par «Manuscrits de Pauline Archange », nous désignons la trilogie formée du roman éponyme, ainsi que des romans Vivre! Vivre! et Les apparences, qui mettent tous en scène le personnage de Pauline Archange. Publiés pour la première fois respectivement en 1968, en 1969 et en 1970 aux Éditions du Jour, ils sont désormais réunis en un seul volume sous le titre Manuscrits de Pauline Archange au Boréal, dans la collection «Boréal compact». 
légitimation qu'elle engage ${ }^{3}$. La notion nous semble d'autant plus intéressante que les Manuscrits de Pauline Archange déploient un vaste réseau thématique autour de l'autorité. Or, si une critique assez virulente des institutions et des personnes qui usent et souvent abusent de leur pouvoir se manifeste au fil des trois romans, la perspective de l'autorité narrative permet de dégager les contradictions d'un récit porté par une voix autoritaire, fragilisée par le discours et le jugement de l'autre. Il semble ainsi, et ce sera le deuxième temps de notre réflexion, que les tensions qui marquent la narration dans la trilogie trouvent leur résolution dans les romans ultérieurs, tels Le sourd dans la ville, Visions d'Anna et, plus tard encore, le cycle inauguré par Soifs. De la voix autoritaire à la voix autorisée, les Manuscrits de Pauline Archange mettraient en scène la difficile conquête de légitimité de l'écriture, à comprendre comme l'atteinte d'une autorité positive de la voix narrative.

\section{UN MONDE AUTORITAIRE}

Globalement, la trilogie de Marie-Claire Blais forme un parcours allant de la petite enfance de Pauline jusqu'au seuil de sa vie d'adulte. Au fil des romans, on voit se manifester le désir d'écrire de Pauline, aspiration qui trouve difficilement à se réaliser dans ce milieu de grande misère économique et sociale. Si l'existence même du récit, assumé par Pauline en narration ultérieure, montre bien qu'elle y est parvenue, il n'empêche que rares ont été les personnes qui l'ont encouragée à suivre cette voie. Le dernier roman se termine d'ailleurs alors que la jeune femme est sans emploi et erre dans la ville revêtue d'un "manteau en guenilles» $(L A, 322)$. Le premier volet, Manuscrits de Pauline Archange, couvre environ deux années de l'enfance : la fillette commence sa vie scolaire et se liera d'amitié avec Séraphine. Le deuxième volet, Vivre! Vivre!, s'ouvre sur le déménagement de la famille dans un nouveau quartier pour ensuite, comme l'a bien noté Réal Girard, se détacher de l'espace de l'école et de la famille au profit de personnages qui occupent d'autres lieux et «contribue[ront] à "l'apprentissage social" ${ }^{4}$ » de Pauline. Au terme de ce roman, l'enfant touche à l'adolescence et s'est mise à écrire. Dans le dernier volet, Les apparences, on verra Pauline obligée par son père à gagner sa vie afin de ne plus être un fardeau pour sa famille: «En septembre, finie l'école, t'as compris» $(L A, 287)$. Ses quelques tentatives de

3 Il est cependant important de préciser que l'autorité narrative ne concerne pas strictement l'instance de narration mais implique d'autres procédés textuels. Pour plus de détails sur la notion d'autorité narrative, on pourra lire: Frances Fortier et Andrée Mercier, «L'autorité narrative en question dans le roman contemporain. Enjeux théoriques et esthétiques d'une notion", Emmanuel Bouju (dir.), L'autorité en littérature, Rennes, Presses universitaires de Rennes, coll. «Interférences», 2010, p. 109-120; de même que Frances Fortier et Andrée Mercier, «L'autorité narrative et ses déclinaisons en fiction contemporaine : Cinéma de Tanguy Viel et Fuir de Jean-Philippe Toussaint", Barbara Havercroft, Pascal Michelucci et Pascal Riendeau (dir.), Le roman français de l'extrême contemporain. Écritures, engagements, énonciations, Québec, Nota bene, coll. "Contemporanéités», 2010, p. 255-275. 4 Réal Girard, «Marie-Claire Blais, écrivain. Les apparences de l'écriture», Livres et auteurs québécois, 1972, p. 369. Pour une présentation plus détaillée de la trilogie, on peut lire, outre l'article de Girard, celui de Nicole Bourbonnais, «Manuscrits de Pauline Archange, trilogie romanesque de Marie-Claire Blais», Maurice Lemire (dir.), Dictionnaire des œeuvres littéraires du Québec, t. IV : 1960-1969, Montréal, Fides, 1984, p. 540-544. 
trouver un emploi se révèlent peu fructueuses, et Pauline devra fréquenter le bureau de chômage. Le cycle romanesque se termine sans que l'on sache comment la jeune femme aura finalement réussi à se débrouiller et à se consacrer à l'écriture tel qu'elle le souhaitait, mais la preuve est faite qu'elle y est arrivée.

Tout au long de ce parcours d'apprentissage, Pauline aura dû faire face à de nombreux obstacles. La misère et tout ce qui l'accompagne - la maladie, l'ignorance, la honte, la violence, le sentiment de la fatalité, etc. - ne laissent aucune place à l'art et à l'écriture et ne leur reconnaissent aucune légitimité. Plus encore, l'enfant et, quelques années plus tard, la jeune fille, se heurte sans cesse à l'autoritarisme des religieuses, des parents et plus largement d'une société qui impose valeurs et modes de vie. Si quelques personnages semblent dotés d'une autorité positive ${ }^{5}$, tels Germaine Léonard, médecin qu'admirent Pauline et son amie Louisette Denis, ou encore Romaine Petit-Page, qui encourage Pauline à écrire, ils perdent presque immanquablement leur lustre et une bonne part de leur crédit: la supériorité morale de Germaine Léonard cède au mépris dont elle use à l'égard d'autrui, la culture et la générosité de Romaine Petit-Page révèlent finalement une "vertu délirante» $(L A, 278)$ et naïve. Dès son arrivée à l'école, Pauline sera confrontée à la "souveraineté brutale (MPA, 41) de mère Sainte-Scholastique et à celle de Dieu, que le catéchisme illustre de façon inquiétante : « une dédaigneuse paupière sous laquelle se réfugiait avec autorité l'œil de Dieu brillant de malice et de dureté» $(M P A, 14)$. Pauline est tout autant soumise à l'autorité de ses parents, «inquisiteurs» $(M P A, 17)$ qui imposent leur «jalouse surveillance» (MPA, 17), et à celle du «tribunal fermé que représentait la famille, groupant autour d'elle les oncles, les cousins, tous ces personnages avares et cruels, débonnaires ou distraits» (MPA, 17). Confiée à son oncle pour les vacances d'été, dans l'espoir qu'elle échappe au climat malsain de la ville et trouve un peu d'appétit, Pauline devra manger de force pendant que des «doigts fébriles comme des pinces » $(M P A, 48)$ lui tiennent la bouche ouverte. Et si sa tante proteste quand l'oncle lui donne des coups au visage, c'est sans jamais mettre en doute le pouvoir absolu des parents: «On peut battre nos enfants, c'est à nous, l'Bon Dieu nous les a donnés, pas les enfants des autres, mon mari...» (MPA, 51)

Face à un tel autoritarisme, quelques personnages tentent bien de se révolter, mais sans parvenir à une véritable émancipation. «Blasphémateur», parce qu'il aime à rire du curé et n'hésite pas à tenir tête à son père, le cousin Jacob finira ses jours interné: "t'as donc pas de cervelle, seulement des dents pour mordre?» (MPA, 50) Le père Benjamin Robert vit bien quelques «moments d'indignation contre toute autorité qui le gênait» $(V V, 159)$, mais il devra expier ses fautes dans «les prisons de mauvais prêtres» $(V V, 160)$, sans compter qu'il porte aussi sur les autres le regard

$$
++
$$

5 Susan Suleiman, auteure de l'ouvrage Le roman à thèse ou l'autorité fictive (Paris, Presses universitaires de France, 1983, 314 p.), distingue ainsi autorité positive et négative: "Dans sa conception négative, l'autorité implique un pouvoir ou une force à laquelle on veut ou on doit résister - c'est ce que j'appellerais: "l'autorité comme cible", c'est-à-dire comme objet de résistance ou de contestation. Dans sa conception positive, l'autorité implique un pouvoir ou une force qui inspire le respect, voire l'admiration - c'est l'autorité comme valeur.» Susan Suleiman et Jean-Louis Jeannelle, «Réflexions sur un parcours. Entretien avec Susan Suleiman », Emmanuel Bouju (dir.), L'autorité en littérature, p. 499. 
inquisiteur - et même plus - de ses désirs illicites : "Ce jour-là, il m’a juste touché la joue et les cheveux» $(V V, 204)$, de révéler pudiquement Pauline. La révolte de Philippe L'Heureux, fils d'un juge « respecté par ses amis, sa famille» $(V V, 173)$, prend la forme du parricide à l'endroit de celui qui avait condamné à mort «un pauvre homme qui, en rentrant chez lui un soir, découragé et ivre, [avait brûlé] la maison où dorm[ai]ent ses neuf enfants et sa femme» $(V V, 173)$; le fils abusant dès lors luimême du pouvoir de la justice. Quant à la protagoniste, qui elle aussi voudrait déchirer «ce voile d'une autorité [qu'elle] n'aimai[t] pas» $(M P A, 56)$ et qui entend bien «se moque[r] désormais de mère Saint-Théophile, de la directrice, de mère SainteScholastique, et même de l'oncle Victorin et du père de Jacob» (MPA, 56), elle parvient mal à se libérer de la dualité victime/coupable. En effet, si elle souffre des multiples formes d'autorité abusive qui s'exercent sur elle, elle n'échappe pas à la dureté, à l'indifférence ou à l'aveuglement qu'elle reproche aux autres. C'est ainsi qu'elle s'éloigne de Séraphine ou de sa grand-mère Josette, qu'elle ne perçoit pas les «désirs violents, inassouvis » $(V V, 156)$ qui habitent sa mère et qu'elle méprise souvent ceux qui subissent le mépris.

\section{DES VOIX AUTORITAIRES}

L'autorité ne se retrouve pas seulement au cœur d'un vaste réseau thématique qui détermine les rapports des personnages, elle marque également la narration du récit. Narratrice autodiégétique, Pauline raconte son difficile apprentissage de la vie et de l'écriture, se remémorant ce qui ne se laisse pas oublier:

Comme le chœur de mes lointaines misères, vieilles ironies que le temps a revêtues du sourire de la pitié (une pitié puant légèrement la mort), les religieuses qui, autrefois, berçaient ma vie de leur cruelle bonté, m'épient encore au grillage d'un cloître perdu dans la fade campagne, sous un ciel mécontent, au bord de la tempête, mais qui accueille comme un encens maladif la plainte de leurs prières, de leurs cantiques au saint sacrement. (MPA, 9)

Cette phrase inaugurale qui semble relever d'une posture en surplomb se laissera toutefois rapidement traverser par de multiples voix et plaintes:

Pauline Archange a désobéi, nous la priverons des restes de notre pain d'hostie... (MPA, 10)

Pauline Archange, priez le Saint-Esprit, comme tout le monde. (MPA, 12)

Vous n'êtes que des bébés, je le dirai à mère supérieure... (MPA, 12)

N'offensez pas Jésus [...], ni dans vos jeux ni dans vos pensées. (MPA, 13)

Ce regard partout vous regarde et vous guette [...]. (MPA, 14) 
Ces paroles rapportées des religieuses rencontreront bientôt celles des parents et de l'entourage :

Mais où étais-tu donc, mauvaise? (MPA, 15)

Tu veux donc tuer ta mère qui est déjà malade? Tu veux me tuer, hein, c'est ça? $(M P A, 15)$

La Pauline, eh ben, elle n'a pas de cœur... (MPA, 18)

On a fait tous les efforts pour c't'enfant-là, et elle nous récompense jamais... (MPA, 18)

Tu vas mourir si tu continues à pas manger comme ça, c'est ça que tu veux, mourir? $(M P A, 23)$

Encadrant souvent de brefs dialogues identifiés par les signes typographiques usuels (saut de ligne et tiret), la narration laisse aussi surgir ces voix au cœur même de son discours. Volontiers sentencieuses, plaintives ou encore violentes, les voix entre guillemets viennent en quelque sorte occuper l'espace de la narration. Si elles ne rompent pas vraiment le propos, elles en heurtent néanmoins le fil par leur ton autoritaire et parfois par leur niveau de langage. Insidieuses, ces voix qui s'immiscent sans cesse dans le discours de la narratrice atteignent l'enfant, et plus tard la jeune fille, qui les reprend à son tour et en vient à les assimiler :

Mais connaissant maintenant ma nature infidèle, je n'osais plus faire de telles promesses. «J'peux pas promettre parce que je suis trop menteuse...»

- Tu mens comme une trompette, disait ma mère, j'ai jamais vu ça quelqu'un qui ment comme ça, tu mens comme tu respires et j'vois ton nez qui bouge comme devant le bon Dieu... (MPA, 57)

La voix en surplomb se voit ainsi constamment ramenée au niveau du sol, subissant le poids de ces paroles dominatrices.

À ces brefs propos qui ponctuent le récit de la narratrice s'ajoutent, dans le deuxième volet de la trilogie, de longs discours rapportés qui en viennent à occuper une place très importante dans l'économie du texte. Contrairement aux courtes incursions de discours direct assumées tout au long des romans par une grande variété de personnages (religieuses, parents, voisinage et entourage, Pauline elle-même, etc.), ces propos sont plus souvent le fait de personnages dont le statut social s'écarte de celui de Pauline: pour l'essentiel, il s'agit de Benjamin Robert, Philippe L'Heureux, Germaine Léonard, Julien Laforêt et Romaine Petit-Page. Vivre! Vivre! se montre ainsi nettement discoureur, envahi par le verbe fluide et les discussions élevées de ceux qui ont le privilège d'être instruits et de provenir d'un milieu aisé (prêtre, fils de juge, médecin, petit-bourgeois philosophe et poète). Si le monde de la diégèse s'élargit au même rythme que Pauline quitte la petite enfance, ces nouveaux personnages ne font 
pas qu'ouvrir les horizons de la jeune fille: ils dominent aussi l'espace du discours et creusent les écarts sociaux. C'est ainsi que la maladresse et l'inculture de Pauline se révèlent encore plus crûment à travers les extraits de ses cahiers d'écriture et les paroles qu'elle s'entend dire avec honte: «Ah! Si je serais un écrivain moi aussi, j'en écriverais donc des livres! C'est facile, vous disez tout ce que vous sentez...» $(V V, 146)$ Figures d'autorité paradoxales, ces personnages ne dialoguent pas avec Pauline mais entre eux, de littérature, de justice, de philosophie ou d'éthique. Si leur parole n'a pas la brutalité de ceux qui ne savent que proférer des ordres et des reproches («Mais la fermeras-tu ta porte de cave de bouche?» [MPA, 21]), elle n'en est pas moins condescendante, sinon méprisante ( Nous serons toujours là pour vous aimer, vous qui n'avez pas de parents» $[V V, 182])$.

Le dernier volet de la trilogie, Les apparences, poursuit l'avancée dans le temps et dans le parcours d'apprentissage de Pauline, mais il délaisse les longs discours rapportés du roman précédent. Les traces de discours direct demeurent sous forme de brefs énoncés, sauf que la narratrice prend davantage à sa charge les propos des uns et des autres, notamment les réflexions de Germaine Léonard. Ce personnage, présent tout au long de la trilogie, a ici un statut particulier. Les affres de sa liaison avec un jeune collègue marié et père de famille sont livrées avec force détails au moyen d'une narration qui se fait omnisciente bien qu'elle soit toujours assumée par Pauline. Ne recourant à aucun des subterfuges traditionnels pour justifier ce savoir et sa profondeur (avoir été témoin ou confident des faits, présenter les scènes de façon hypothétique, etc.), la narration prend ses libertés à l'égard de la vraisemblance pragmatique ${ }^{6}$, comme elle le faisait d'ailleurs dans le volet précédent en livrant «textuellement» de longs entretiens, mais cette fois elle le fait de manière beaucoup plus marquée puisque ce sont les pensées intimes, et souvent même inconscientes, de Germaine Léonard et de son amant qui sont révélées:

elle qui était si présomptueuse, souvent si rigide dans ses jugements, condamnait la cérébralité de Pierre Olivier, son ténébreux orgueil, sans reconnaître ces fautes en elle-même, car elle était incapable d'admettre la supériorité d'esprit que Pierre Olivier avait sur elle, et malgré les éclairs de modestie qui traversaient son cœur elle n'osait pas même penser : "Nous sommes égaux» (cette simple égalité eût représenté pour elle une blessure à son orgueil) [...]. (LA, 249; nous soulignons.)

Ce récit de pensée, qui ne se limite pas à l'introspection de la propre conscience de Pauline mais s'étend à la « voix intérieure» $(L A, 253)$ d'autres personnages ${ }^{7}$, distingue

6 La vraisemblance pragmatique concerne les conditions d'acceptabilité de l'acte de narration, plus particulièrement le mode d'information du narrateur et les circonstances de l'énonciation. Voir Cécile Cavillac, «Vraisemblance pragmatique et autorité fictionnelle», Poétique, n 101, février 1995, p. 24.7 S'il ne signale pas cet inhabituel usage de la narration omnisciente dans l'article qu'il consacre à la trilogie, Réal Girard évoque tout de même certains blâmes que l'on a pu adresser à l'œuvre de Marie-Claire Blais et qui pourraient concerner ce genre de libertés: «il y a les doctes critiques qui reprochent à Marie-Claire Blais "de nombreuses naïvetés", "de graves fautes de psychologie et de technique", "des faiblesses flagrantes de style" ». ("Marie-Claire Blais, écrivain», p. 365; les sources de ces critiques ne sont toutefois pas précisées.) Jacqueline Viswanathan note, quant à elle, ces «curieuses 
Les apparences des autres romans de la trilogie. Sur le plan de l'autorité narrative, on note dès lors une affirmation beaucoup plus nette des pouvoirs d'investigation et d'appropriation de la narratrice, comme si, au fil des romans, le parcours de formation de Pauline se menait tant dans le monde représenté que dans le travail de la narration. Traversé par les paroles et les jugements des autres, le récit se transforme jusqu'à atteindre et démystifier les figures d'autorité, surtout celle de Germaine Léonard, médecin tant admirée par Pauline, mais capable aussi du plus cruel mépris à son endroit, et qui se verra finalement soumise à son tour au regard sans complaisance de la narratrice ${ }^{8}$.

\section{LES TENSIONS DE LA NARRATION}

Peut-on conclure pour autant à une résolution de la quête de Pauline? De fait, il semble bien que la jeune fille ait pris sa revanche sur ceux qui ont douté d'elle ou fait entrave à son projet de vie. Et même si la posture en surplomb de la narratrice connaît de nombreux aléas au fil du récit - traversée par des voix sentencieuses ou écartée des échanges savants -, elle n'en arrive pas moins à s'immiscer dans l'intimité profonde de Germaine Léonard pour ainsi déconstruire cette figure d'autorité admirée en même temps que crainte. Au terme du récit, et bien que la jeune fille n'ait pas achevé son parcours de formation et que sa situation se montre franchement précaire, se manifeste le premier signe d'une véritable connivence entre Pauline et André Chevreux, étudiant aux origines aussi modestes que les siennes. Alors que la tempête fait rage et que la voix du père se fait de nouveau entendre ("Ah! Je suis bien fatigué, c'est comme si mon cœur ne battait plus au bout de sa corde! » [LA, 322]), une entente réelle semble pour une fois possible:

Il s'approcha de la fenêtre givrée et me sourit. Il y avait dans ce sourire une telle amitié, une si tendre vaillance que je sentis soudain renaître mon courage et, emportant cette vision dans la tempête, je courais en pensant avec joie : «C'est lui... l'ange de Dürer, je l'ai vu, enfin!» (LA, 322)

Figure de la création, et plus encore figure de la rencontre du génie et du travail quotidien, l'ange de Dürer (dont une religieuse avait accroché une reproduction sur le

$$
+++
$$

anomalies » des Manuscrits de Pauline Archange qu'elle considère comme « une tentative intéressante pour repousser les limites de la narration à la première personne au-delà de la complaisance de l'introspection ». Jacqueline Viswanathan, «"Cette danse au fond des cœurs." Transparence des consciences dans Le sourd dans la ville et Visions d'Anna de M.-C. Blais», Canadian Literature, nº 111, hiver 1986, p. 89.8 C'est aussi le cas de Romaine PetitPage, qui sera l'objet de l'ironie de la narratrice et de ses jugements sévères. L'omniscience touche ici son mari, Louis, révélant sa lassitude à l'égard de sa femme et certains secrets: "[...] Louis qui, ennuyé de séduire une femme toujours délirante de vertu, masquant sans cesse ses désirs de subtiles et provocantes piétés, trouvait en Romaine ce qu'il avait parfois aimé en d'autres femmes, le charme de ces prostituées avec lesquelles il avait trahi sa fiancée à Paris (ne pouvant plus attendre une récompense tant de fois promise et retirée par la catholique Romaine)» (LA, 278). 
mur de la classe ${ }^{9}$ ) devient tout à coup une réalité incarnée par André Chevreux, capable de donner à Pauline la force de poursuivre sa quête d'écriture. En effet, à travers cette incarnation, l'art n'est plus la paresse à laquelle les parents de Pauline ont toujours ramené son désir d'écrire. Il n'est pas non plus détaché de la réalité, comme le pensent Julien et Romaine Petit-Page. Il est méditation et labeur de l'ouvrier.

Pourtant, si Pauline trouve enfin l'image et la personne en mesure de représenter son être véritable et ses aspirations, si le sourire d'André Chevreux échappe aux rapports de mépris et de brutalité auxquels elle semblait condamnée, le récit n'en reste pas moins porté tout au long par de fortes tensions qui, sur le plan de la narration en particulier, ne paraissent pas totalement dénouées. Victime du regard des autres, toujours prêts à la juger mais incapables de franchir les apparences, Pauline cherche à atteindre la vérité. Les voix autoritaires qui ponctuent le récit sont toutes entachées de ce regard aveugle, le plus souvent impitoyable, qui ramène sans cesse à la honte. Quand elles se mettent à discourir du haut de leur savoir, elles ne parviennent pas davantage à révéler ce que sont les êtres, ni à établir un véritable dialogue, ne faisant qu'accentuer l'écart social. Or, si l'omniscience permet de traverser les apparences, elle le fait avec une insistance et une sévérité qui, à bien des égards, ressemblent à l'autoritarisme subi et dénoncé par Pauline. Cette attitude, qui semble heurter l'idéal éthique et esthétique qui anime la quête de Pauline, se manifeste à travers les figures du regard et de la création ${ }^{10}$. Engagés dans une discussion sur L'idiot de Dostoïevski, Germaine Léonard et son amant, Pierre Olivier, confrontent leurs visions respectives et antithétiques du roman, la première y voyant à l'œuvre «un regard puissant et sans complaisance», celui de «l'intervention noble, détachée, d'une conscience d'écrivain» (LA, 262), alors que le second défend plutôt «la force, l'irrationnelle violence qui ont saisi l'écrivain quand il pénétrait la vie d'un tel personnage» (LA, 262-263). Entre la distance «sans complaisance » et "l'irrationnelle violence», où se situe la narration des Manuscrits de Pauline Archange? Arrive-t-elle à poser le regard juste qui ne serait ni noble ni avide? On peut en douter.

Les marques fréquentes d'ironie, de plus en plus nettes dans le dernier volet de la trilogie, se montrent de fait fort peu complaisantes. En font principalement les frais les personnages jadis admirés par Pauline, tels Germaine Léonard, Romaine PetitPage et le jeune Julien Laforêt, tournés en ridicule par l'instance narrante. S'il est vrai que les voix des uns et des autres s'immiscent souvent dans le récit de la narratrice au point d'occuper l'espace du discours, il est aussi vrai qu'elles peuvent se retourner contre elles-mêmes par la naïveté ou la suffisance de leurs propos. Alors que le personnage de Pauline adolescente envie la culture et l'assurance de Julien («En tout cas, moi j'serai pas une cruche comme grand-maman qui savait même pas lire et qui

$$
++
$$

9 La description de la toile de Dürer, Melancholia, apparaît à la page 290 de la trilogie. 10 On observe en effet un vaste réseau thématique et figuratif qui se déploie autour du regard et de la création. Le regard se montre d'ailleurs étroitement lié à la figure de l'autorité aussi bien négative que positive, selon qu'il juge et blesse les personnages (les exemples en sont nombreux, mais on peut penser à la mère de Clara dont le regard acharné est particulièrement cruel $[V V, 138]$ ) ou qu'il aspire à la vérité (Émile, petit frère infirme de Pauline et que l'on dit aveugle, correspond à cet idéal [MPA, 98, 105-106]). 
signait son nom avec une croix, c'est de la vraie honte à soixante-dix ans! Julien Laforêt, y a appris à lire et à écrire quand y avait quatre ans, et tout seul, à part ça » $[V V, 194])$, la narratrice se montre plutôt mordante à l'égard du garçon en rapportant simplement ses paroles:

Je marchais silencieusement à ses côtés mais il s'écria: - Taisez-vous, Pauline, les femmes devraient toujours se taire. Quand j'écoute notre amie Romaine, j'ai toujours faim, je veux manger des pommes, j'aime la vie terrestre, moi! Mais vous, Pauline, écrivez des mauvais poèmes mais je vous défends de les lire à haute voix : c'est trop pénible. Vous bâillez? Reposez-vous donc ici, sous cet arbre, je vous parlerai de Platon. (VV, 198-199)

Les jugements et les explications de la narratrice ponctuent par ailleurs le récit sans faillir, souvent sous forme de parenthèses et avec une présence particulière dans le dernier roman. Les apparences se voient ainsi percées sans aucune indulgence:

Germaine Léonard avait l'habitude de dominer ceux qu'elle aimait (croyant être aimée pour sa force, elle ne montrait souvent que sa maladresse, son impuissance) [...]. $(L A, 249)$

Souvent, après avoir accablé d'injures un écrivain qui était jeune (donc menaçant pour elle), Germaine Léonard s'attardait avec émotion «à l'ombre généreuse des œuvres du passé» [...]. (LA, 252)

Si l'autorité narrative oscille au fil du récit entre plusieurs voix et plusieurs postures, elle finit par rejoindre ce regard souverain qui, à bien des égards, se montre semblable à «l'œil de Dieu brillant de malice et de dureté» $(M P A, 14)$ que cherchait tant à défier Pauline.

\section{UNE VOIX AUTORISÉE}

Si Jacqueline Viswanathan considérait, en 1986, Le sourd dans la ville et Visions d'Anna comme l'aboutissement de l'œuvre de Blais ${ }^{11}$, ces deux romans nous apparaissent maintenant plutôt comme la mise en place d'un style nouveau ${ }^{12}$ qui est devenu la marque de commerce de l'écrivaine et qui s'est définitivement fixé dans le cycle romanesque amorcé par Soifs. Ce style, qui se caractérise par la continuité - textes sans

$$
+++
$$

11 Jacqueline Viswanathan, "Cette danse au fond des cœurs" », p. 93. Michel Biron et Nathalie Roy utilisent aussi le terme d' "aboutissement», mais pour caractériser les trois premiers romans de la série inaugurée par Soifs. Michel Biron, «De la compassion comme valeur romanesque», Voix et Images, vol. XXXI, n 1, automne 2005, p. 141, et Nathalie Roy, De l'ironie romantique au roman contemporain. L'esthétique réflexive comme philosophie dans la trilogie Soifs de Marie-Claire Blais, thèse de doctorat, Montréal, Université du Québec à Montréal, 2007, f. 156. 12 Pour caractériser Le sourd dans la ville et Visions d'Anna, Élène Cliche parle notamment de «nouvelle rythmique de la phrase». Élène Cliche, «Un rituel de l'avidité», Voix et Images, vol. VIII, n 2, hiver 1983, p. 230. 
paragraphes, phrases très longues, peu de ponctuations fortes, focalisation multiple qui « glisse» d'une conscience à l'autre - , peut sembler a priori n'avoir rien en commun avec l'écriture des Manuscrits de Pauline Archange. Pourtant, à plusieurs égards, Le sourd dans la ville, Visions d'Anna et Soifs paraissent répondre à la trilogie en résolvant les tensions qui en émergent. Cette résolution est notamment le fait d'une voix narrative qui s'adoucit et de la naturalisation des transitions entre les consciences.

C'est une voix très discrète qui prend en charge la narration du Sourd dans la ville, de Visions d'Anna et de Soifs. Contrairement aux Manuscrits, ces romans présentent un narrateur hétérodiégétique, donc extérieur à l'histoire racontée et qui, en outre, prend très peu de place dans le récit. Il s'efface en effet devant l'exposition de la vie intérieure des personnages et n'intervient que très rarement, comme le remarque Viswanathan: "Ce qui frappe dans [...] Le sourd dans la ville, c'est le glissement d'une conscience à l'autre sans l'intervention d'un passage commentatif, descriptif ou narratif ou la transition d'un dialogue ${ }^{13}{ }^{\prime}$. Ainsi, le texte est constitué pour l'essentiel de fragments de monologues narrativisés - surtout, mais aussi de psycho-récits et de monologues rapportés, comme le montre bien Nathalie Roy ${ }^{14}$ - qui représentent la pensée des personnages sur le mode du stream of consciousness et entre lesquels le narrateur ne se fait guère entendre. En effet, celui-ci mêle plutôt sa voix à celles des personnages, si bien qu'il devient souvent impossible de lui attribuer exclusivement l'origine d'une proposition ${ }^{15}$. L'extrait suivant montre bien la dualité propre au monologue narrativisé :

La terre était craquelée et rugueuse autour des cactus, pensait le pasteur, c'était le signe d'une proche sécheresse, et qu'avait-il vu dans le temple pendant l'office, ces filles fardées de rouge, les siennes, les lèvres boursouflées, c'était l'enflure du désir, de l'appétit sexuel, [...] Dieu avait créé la sécheresse et les filles, ces démons qui insultaient le Seigneur, [...] il se souvint que le péché était dans sa maison [...]. (SO, 30)

Tandis que la voix narrative renvoie ici au pasteur à l'aide de la troisième personne, c'est la perspective de ce dernier qui est privilégiée dans l'énoncé, ce qui est rendu visible notamment par le vocabulaire employé et par la morale véhiculée. Dans Le sourd dans la ville, Visions d'Anna et Soifs, le rôle du narrateur se limite donc souvent à assurer les transitions entre les personnages et à rappeler - entre autres par l'utilisation du verbe "penser", qui revient constamment - de quelle conscience origine la perspective retenue.

Pourtant, même si cette voix est effacée, elle emplit paradoxalement le texte de sa présence: il y a très peu de discours directs, et la narration à la troisième

$$
++
$$

13 Jacqueline Viswanathan, «Échanger sa vie pour une autre. Focalisation multiple dans Mrs. Dalloway et Le sourd dans la ville», Arcadia. Zeitschrift für Vergleichende Literaturwissenschaft, vol. XX, n² 2, 1985, p. 182.14 Nathalie Roy, De l'ironie romantique au roman contemporain, f. 175-182. 15 Ce serait, selon Dorrit Cohn, l'une des caractéristiques du monologue narrativisé que de confondre les deux énonciations: "[Le monologue narrativisé] imite le langage dont se sert un personnage lorsqu'il se parle à lui-même, mais il soumet ce langage à la syntaxe dont se sert le narrateur pour parler de ce personnage; il superpose ainsi l'une à l'autre les deux voix que les autres formes distinguent nettement. » Dorrit Cohn, La transparence intérieure. Modes de représentation de la vie psychique dans le roman, traduit de l'anglais par Alain Bony, Paris, Éditions du Seuil, coll. «Poétique», 1981, p. 127. 
personne, maintenue tout au long du récit, contraint à affirmer que l'énonciation de ces romans est bel et bien le fait d'un narrateur hétérodiégétique. Il y a donc une certaine univocité ${ }^{16}$ qui se dégage de ces textes; $c^{\prime}$ est en effet une «parole unique ${ }^{17}$ » qui prend en charge l'histoire et qui nous fait visiter les diverses consciences. Tout se fond dans une narration homogène ${ }^{18}$ qui ne cherche pas à mimer la voix de chacun des personnages, mais qui, plutôt, en représentant «un en deçà de la pensée verbalisée ${ }^{19}$ ", laisse tomber toute marque linguistique individuelle: «les traces $\mathrm{d}$ 'idiolectes sont assez rares même dans les discours directs ${ }^{20}$ ». L'autorité de la voix narrative est donc différemment construite: celle-ci n'entre plus en concurrence avec d'autres voix, ne cherche plus à affirmer sa légitimité. Son ironie et ses jugements sont à la fois moins présents et plus difficilement perceptibles que dans la trilogie. C'est donc une autorité beaucoup plus subtile, et par le fait même d'autant plus efficace, qui se met en place: il s'avère en effet difficile de contester la crédibilité de cette narration qui s'investit si peu dans le récit tout en assurant une médiation rigoureuse entre les personnages.

\title{
PERCER LES APPARENCES
}

$\mathrm{Si}$, en comparaison avec les Manuscrits de Pauline Archange, la voix narrative des romans ultérieurs semble avoir trouvé, dans un style homogène, une solution aux tensions des voix multiples et autoritaires qui s'entrecroisent, le système de focalisation $^{21}$, en s'assouplissant, permet de voyager entre les consciences d'une façon beaucoup plus acceptable aux yeux du lecteur. À l'omniscience de Pauline succède, dans les romans suivants, un jeu de focalisations enchâssées qui, combiné à l'impression de cohésion et de continuité qui se dégage du texte, tend à naturaliser les transitions entre les consciences. En voici un exemple, parmi tant d'autres:

\begin{abstract}
Nous serons dans le désert, pensait Mike, très loin d'ici d'eux tous, l'indigo bush qui guérit, la lavande et le miel, murmurait-il à Jojo en lui caressant les cheveux, et Florence voyait cette longue main pâle de Mike qui reposait sur la tête de sa sœur, que de promesses sensibles offrait cette main. $(S D V, 176)$
\end{abstract}

Ainsi, comme on le voit ici, l'entrée dans la vie intérieure des personnages est marquée d'une certaine fluidité. C'est d'ailleurs le terme de «glissement» qui, opposé à celui

$$
++
$$

16 Il peut certes sembler paradoxal de parler d' «univocité » pour des textes qui présentent la perspective de tant de personnages différents. Nous nous référons cependant ici à l'analyse de Lucie Bourassa qui oppose voix et regard en distinguant la polyphonie de Faulkner (où s'entremêlent plusieurs voix) de la polyscopie de Blais (où ce sont plusieurs regards qui s'entremêlent). Lucie Bourassa, «Focalisation et vocalisation. La conscience narrative», Protée, vol. XVI, $\mathrm{n}^{\text {os }}$ 1-2, hiver-printemps 1988, p. 93-103. 17 Ibid., p. 95.18 Viswanathan parle en effet d'«homogénéité stylistique», et Bourassa d' "homogénéité phrastique». Jacqueline Viswanathan, "Échanger sa vie pour une autre», p. 190, et Lucie Bourassa, «Focalisation et vocalisation», p. 95.19 Nathalie Roy, De l'ironie romantique au roman contemporain, f. 170. 20 Jacqueline Viswanathan, «Échanger sa vie pour une autre», p. 190. 21 Pour une description plus détaillée de la focalisation dans Le sourd dans la ville, voir Jacqueline Viswanathan, «Échanger sa vie pour une autre», et Lucie Bourassa, «Focalisation et vocalisation». 
de "rupture», ressort des études critiques pour caractériser les transitions entre les consciences dans Le sourd dans la ville ${ }^{22}$. Ce procédé est d'autant plus frappant que la diégèse réunit des personnages qui interagissent peu: ils ne se connaissent pas tous, ils se parlent peu, ce qui n'empêche pourtant pas la perspective du récit de voyager constamment d'une conscience à l'autre. À propos du Sourd dans la ville, Viswanathan remarque d'ailleurs, à juste titre, que « [c]e qui caractérise [le roman], c'est la force et l'importance des rapports, sans doute compensatoires, qui se créent avec des inconnus ${ }^{23}$ ", rapports qui se tissent, justement, par l'entremise du texte qui réunit les personnages non pas sur le plan des faits et des événements, mais plutôt par le truchement du mode de focalisation.

Souvent ténus, les liens entre les personnages, qui conduisent à un changement de sujet focalisateur, peuvent se limiter à un simple regard, comme l'a bien montré Bourassa ${ }^{24}$, qui nomme "polyscopie» ce jeu de regards enchâssés qui caractérise notamment la focalisation du Sourd dans la ville: «un regard voit par les yeux des autres [...]. Le personnage est vu ou voyant et son regard devient lui-même objet de regard ${ }^{25}$ ». La scène finale du roman en donne un excellent exemple : « [Mike] regardait Florence, sans bouger, [...] bientôt elle ne les verrait plus, elle ne verrait plus Mike, [...] ce n'était qu'une silhouette dans le brouillard, qu'un fantôme, peut-être, elle ne les voyait plus, elle avait fermé les yeux " (SDV, 187). Le regard a ainsi conservé toute l'importance qu'il pouvait avoir dans les Manuscrits de Pauline Archange : il est ce par quoi le « désir de pénétration et de possession de la conscience de l'autre ${ }^{26}$ » se réalise. Succédant aux dons d'omniscience de Pauline, la fluidité des transitions et l'enchâssement des regards, qui caractérisent les romans ultérieurs, marquent ainsi une nouvelle façon de percer les apparences des êtres.

\section{LE CHEUR DES CONSCIENCES}

Malgré quelques différences stylistiques marquées à partir du Sourd dans la ville, la plupart des critiques s'entendent pour voir dans l'œuvre de Marie-Claire Blais une certaine continuité ${ }^{27}$; l'analyse des Manuscrits de Pauline Archange que nous proposons ici s'inscrit dans le même esprit. Il nous semble en effet que cette trilogie, somme toute très peu étudiée, contient déjà, en germes, les techniques narratives des romans à venir. Confrontée à l'univers tyrannique qui l'entoure, la jeune Pauline, qui rêve d'écrire sa vie, est en quête d'autorité : elle cherche à poser sa voix à travers un tissu

$$
++
$$

22 Voir notamment Jacqueline Viswanathan, «Échanger sa vie pour une autre», p. 182, et Martin Herden, «Le monologue intérieur dans The Sound and the Fury de William Faulkner et Le sourd dans la ville de Marie-Claire Blais», Voix et Images, vol. XIV, n 3, printemps 1989, p. 495. 23 Jacqueline Viswanathan, "Échanger sa vie pour une autre», p. 191. Notons que cette question des rapports avec des inconnus apparaissait déjà dans les Manuscrits de Pauline Archange: Pauline essaie, avec plus ou moins de succès, de nouer des liens étroits avec, par exemple, Germaine Léonard ou encore Romaine Petit-Page. 24 Lucie Bourassa, "Focalisation et vocalisation ", p. 94-97. 25 Ibid., p. 94. 26 Jacqueline Viswanathan, "Cette danse au fond des cœurs" », p. 87.27 Voir, par exemple, Jacqueline Viswanathan, " "Cette danse au fond des cœurs" », p. 93 ; Michel Biron, «De la compassion comme valeur romanesque», p. 141; et Élène Cliche, «Un rituel de l'avidité», p. 230. 
de discours autoritaires. Dans cette poursuite de légitimité, elle en vient à adopter les comportements de son entourage; en tant que narratrice, Pauline se montre très autoritaire : elle juge, commente et se permet des incursions dans les pensées des autres, autant d'interventions qui envahissent le récit des Manuscrits de Pauline Archange. Ce sont, selon nous, les romans ultérieurs qui régleront ces tensions de la narration en mettant en scène une voix plus effacée et en proposant une façon moins brutale de pénétrer la conscience des autres.

Cette volonté de percer les apparences et d'atteindre la conscience de l'autre est d'ailleurs, selon Viswanathan, « une recherche fondamentale de l'œuvre de MarieClaire Blais ${ }^{28}$ ». Elle s'accompagne notamment d'un mouvement de décentrement du sujet déjà visible à l'intérieur des Manuscrits de Pauline Archange — la vie de Pauline prend beaucoup moins de place dans les deuxième et troisième tomes de la trilogie que dans le premier - et que l'on peut suivre à travers toute l'œuvre ultérieure: le sujet énonciateur s'efface, laissant place à une multitude de sujets focalisateurs qui forment une mosaïque où aucun ne parvient réellement à s'imposer. Ces sujets démultipliés fondent néanmoins une sorte de «communauté des consciences » qui est le fait de la forme même du récit:

la forme qui concrétise l'aspiration vers l'unité et l'ordonnance universellement englobante se doit de refléter chez Blais le fait que cette aspiration, sur le plan philosophique, est résolument supra-individuelle, visant [...] la représentation d'une conscience universelle. [...] On peut penser [...] que la forme narrative, pour atteindre sa cohésion, doit justement déjouer une linéarité temporelle et diégétique qui sépare pour atteindre dans sa visée synthétique une sorte de paradoxale «identité», une identité que l'on pourrait qualifier de plurielle. Les licences stylistiques à l'échelle de la phrase même, avec sa structure circulaire, sa longueur qui cumule une pluralité de perspectives et ses répétitions formelles, paraissent refléter cette volonté de courtcircuiter les effets de la linéarité de l'ordre langagier, qui sépare toujours le sujet de l'objet, et le sujet des autres sujets ${ }^{29}$.

Ainsi, c'est le caractère continu du style de Blais qui est à l'origine des rapprochements entre les personnages ${ }^{30}$. Toutes ces vies intérieures s'unissent pour donner naissance au récit; à la manière d'un chœur, l'énonciation se déploie entre unité et pluralité. La figure du chœur a donc effectué une importante migration: image récurrente dans les Manuscrits de Pauline Archange, c'est sur le plan formel qu'on la retrouvera dans les romans ultérieurs. Le récit est en effet placé sous la responsabilité d'un chœur des consciences qui peut rappeler, à maints égards, ce que Susan Lanser nomme, dans son ouvrage Fictions of Authority ${ }^{31}$, une " voix collective» ("communal voice»), c'est-à-dire soit un ensemble de «voix qui partagent l'autorité narrative ${ }^{32}$ » soit «la voix d'un

28 Jacqueline Viswanathan, «Cette danse au fond des cœurs», p. 87. 29 Nathalie Roy, De l'ironie romantique au roman contemporain, f. 183. Roy souligne. 30 Roy note d'ailleurs que, chez Blais, «l'abolition de la distance [...] est davantage le fait des stratégies narratives». Ibid., f. 213. 31 Susan Sniader Lanser, Fictions of Authority: Women Writers and Narrative Voice, Ithaca, Cornell University Press, 1992, 287 p. 32 Ibid., p. 21. Nous traduisons. 
simple individu manifestement autorisé par sa communauté ${ }^{33}$ »; en somme, un chœur en accord avec l'idéal social et esthétique que cherchent à atteindre les Manuscrits de Pauline Archange.

33 Ibid. Nous traduisons. 


\section{IS T E DES S I G LES}

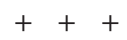

Afin d'alléger le système de notes, les références aux œuvres de Marie-Claire Blais mentionnées dans ce dossier seront indiquées par des sigles, suivis du folio, et placées entre parenthèses dans le texte:

Augustino et le chœur de la destruction (ACD), Montréal, Boréal, 2005, 302 p.

Dans la foudre et la lumière (DFL), Montréal, Boréal, 2001, 251 p.

Mai au bal des prédateurs (MBP), Montréal, Boréal, 2010, 322 p.

Manuscrits de Pauline Archange (PA), Montréal, Stanké, 1988 [1968], 223 p.

Manuscrits de Pauline Archange (MPA) [1968], Vivre! Vivre! (VV) [1969], Les apparences (LA) [1970], Montréal, Boréal, coll. «Compact», 1991, 324 p.

«Marie-Claire Blais», Catherine Morency (dir.), La littérature par elle-même (LPE), Québec, Nota bene, 2005, p. 17-21.

Naissance de Rebecca à l'ère des tourments (NR), Montréal, Boréal, 2008, 296 p.

Parcours d'un écrivain. Notes américaines (PDE), Montréal, VLB éditeur, 1993, 222 p.

Soifs (SO), Montréal, Boréal, 1995, 314 p.

Soifs (S), Montréal, Boréal, coll. «Compact», 1997 [1995], 320 p.

Une saison dans la vie d'Emmanuel (SVE), Montréal, Éditions du Jour, 1968 [1965], $128 \mathrm{p}$. 